\title{
Abandono del tratamiento en adictos a la cocaína
}

\section{Treatment dropout in cocaine addicts}

\author{
| Emilio Sánchez-Hervás*; Roberto Secades-VilLa**; \\ Francisco José Santonja Gómez ${ }^{\star \star *}$; Francisco \\ Zacarés Romaguera* ${ }^{* *}$ Olaya García-RodríGuez ${ }^{* * * *}$ \\ Elena Martin Yanez ${ }^{* * * * *}$, María Calatayud

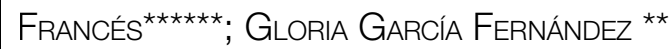

UCA Catarroja. Consellería de Sanitat. Valencia. "Universidad de Oviedo.

"*'Centro Estema. Universidad Miguel Hernández. Alicante.

"*tuniversidad de Barcelona.

UCA Campanar. Consellería de Sanitat. Valencia.

UCA Alzira. Hospital la Ribera. Consellería de Sanitat. Valencia

Enviar correspondencia a:

Emilio Sánchez Hervás

Unidad de Conductas Adictivas-Centro de Salud.

Avd. Rambleta, 63. 46470 Catarroja. Valencia. España

E-mail: esh455k@gmail.com

\section{RESUMEN}

La alta tasa de abandonos de los programas de tratamiento es uno de los problemas más graves en el ámbito de las drogodependencias. La identificación temprana de predictores de riesgo de abandono puede proporcionar información útil sobre las necesidades especificas de tratamiento de este tipo de pacientes. El objetivo de este estudio fue identificar predictores de la finalización prematura de un programa de tratamiento ambulatorio para adictos a la cocaina. El estudio se realizó en varias unidades asistenciales públicas de una ciudad española. Los participantes fueron 91 adictos a la cocaina admitidos para ser tratados de su adicción, evaluados mediante entrevista y varias medidas de autoinforme. Para identificar las variables predictivas se utilizó un análisis factorial, un análisis cluster y un análisis CHAID.

Las variables que obtuvieron capacidad predictiva fueron las puntuaciones en el MAST, y las puntuaciones compuestas de alcohol y drogas del EuropASI. Estas tres variables quedaron agrupadas en un mismo factor al que se denominó gravedad adictiva. El análisis predictivo mostró que este factor tenía una cierta capacidad de predicción del abandono, pero que no era totalmente determinante. Los resultados apuntan hacia la conveniencia de detectar en la admisión a los pacientes que pudieran requerir una mayor preparación motivacional, mayor control e intensidad en el tratamiento, o la prestación de intervenciones coadyuvantes.

\section{ABSTRACT}

The high rate of dropout from treatment programs is one of the most serious problems in the field of drug dependence. Early identification of predictors of dropout risk can provide useful information on the specific treatment needs of these types of patient. The aim of this study was to identify predictors of premature cessation of an outpatient treatment program for cocaine addicts. The study was carried out at public healthcare units in a Spanish city. Participants were 91 cocaine addicts admitted for treatment for their addiction, assessed by means of interview and various self-report measures. For identifying predictive variables the researchers used a factor analysis, a cluster analysis and a CHAID analysis.

The variables that obtained predictive capacity were the MAST scores and the combined alcohol and drugs scores on the EuropASI. These three variables were grouped in a single factor which was called addictive severity. The predictive analysis showed that this factor had some degree of capacity for the prediction of dropout, but that it was not completely determinant. The results suggest the advantageousness of detecting at the admission stage those patients who might require more attention to their motivational aspects, greater treatment control and intensity, or the provision of complementary interventions.

Key words: cocaine, treatment dropout, addiction severity 


\section{INTRODUCCIÓN}

os intentos por mejorar los resultados del tratamiento de las personas con problemas relacionados con el abuso y la dependencia a la cocaína han generado en los últimos años una creciente investigación sobre la eficacia y la efectividad de los programas de tratamiento. Recientes metaanálisis señalan que las tasas de cumplimiento de los programas de tratamiento en drogodependencias ofrecen unos resultados moderados, y se sitúan en torno al cincuenta por ciento'. Las tasas de cumplimiento de los programas para dependientes a cocaína son aún menores ${ }^{2}$. Por ello, uno de los principales objetivos de los tratamientos es el de retener a los pacientes, puesto que ello mejora los resultados a largo plazo.

Desde una perspectiva clínica algunas evidencias sugieren que los individuos que abandonan prematuramente los programas de tratamiento tienen un mal pronóstico. Menos de treinta dias en tratamiento se ha relacionado con mayores tasas de recaída ${ }^{3,4}$; mientras que una permanencia de más de seis meses se ha asociado a reducción del consumo, de la actividad legal y aumento en el tiempo de empleo ${ }^{5}$. Mayor tiempo en tratamiento también se ha asociado a una reducción del consumo en usuarios de "crack" ${ }^{6}$, aunque este tipo de pacientes presentan retenciones más bajas por ser más difíciles de tratar?.

Puesto que los problemas relacionados con la dependencia a la cocaína son heterogéneos, diferentes factores podrían explicar las razones por las que los pacientes deciden abandonan antes de finalizar los tratamientos. Los estudios sobre los factores relacionados con la retención en los tratamientos para la adicción a la cocaína suelen incluir dos tipos de variables, las relacionadas con los pacientes y las relacionadas con el tratamiento. Dentro de los primeros, se ha encontrado que variables como la psicopatología asocia$d a^{8}$, la edad, el empleo o el estado civil ${ }^{9}$, el sexo ${ }^{10}$, la satisfacción con el tratamiento ${ }^{11}$, las deficiencias cognitivas ${ }^{12} 0$ la impulsividad y la búsqueda de sensaciones ${ }^{13}$ pueden estar asociadas a la retención. Los estudios sobre las variables relacionadas con el tratamiento sugieren que la mayor oferta en los servicios ${ }^{14,15}$, el empleo de servicios psiquiátricos ${ }^{16,17}$, una mayor intensidad en el tratamiento ${ }^{18}$, la alianza terapéutica $^{19}$, la participación en grupos de autoayuda ${ }^{20}$, el nivel de implicación en las tareas de terapia entre sesiones ${ }^{21}$, el uso de disulfiram ${ }^{22}$, o el empleo de incentivos ${ }^{23}$ pueden disminuir la retención e incrementar la abstinencia. La confluencia de diversos factores y variables, y su interacción dinámica a lo largo de los procesos que operan durante el tratamiento, determinan una mayor o menor probabilidad de abandono.

El objetivo de este estudio fue el de entender mejor como todos estos factores influyen en el resultado del tratamiento entre los individuos con dependencia a la cocaína. Para ello, se desarrolló un modelo matemático que agrupaba y clasificaba a los pacientes en función de unos perfiles concretos, con el fin de analizar si estos perfiles eran determinantes del abandono del tratamiento. Los datos resultantes podrían proporcionar información útil sobre las necesidades especificas de tratamiento en este tipo de pacientes.

\section{MÉTODO}

\section{Participantes}

El estudio se realizó en tres unidades de conductas adictivas; centros de tratamiento ambulatorio públicos dependientes de la Consellería de Sanitat de Valencia (España), durante los años 2007-2008. Los criterios de inclusión fueron ser mayor de edad y cumplir criterios para el diagnóstico de dependencia a la cocaína según el DSM-IV-TR ${ }^{24}$. Los criterios de exclusión fueron presentar trastornos psicopatológicos graves (p.ej. esquizofrenia, demencia), o barreras linguísticas que impidieran cumplimentar correctamente los instrumentos de evaluación. La muestra final estuvo compuesta por 91 pacientes admitidos para tratar sus problemas de adicción a la cocaina. La media de edad fue de 31,3 años. El $86,8 \%$ eran hombres, el $45,1 \%$ solteros y el $77,5 \%$ estaban activos laboralmente. Presentaron un promedio de 5,76 criterios de dependencia a la cocaína según criterios de la American Psychiatric Association, y una antigüedad en el consumo de cocaína de 10,4 años. La vía de administración predominante era la nasal (94,4\% de los casos), el 10,1\% tenían antecedentes penales y el $14,6 \%$ padecía problemas médicos crónicos.

\section{Instrumentos}

Se utilizó una entrevista de elaboración propia para recoger la información sobre las características sociodemográficas, y se administró el cuestionario de criterios de dependencia a la cocaína del DSM-IV-TR ${ }^{24}$. Las medidas relacionadas con el funcionamiento psico-social y la severidad de la adicción se obtuvieron con las puntuaciones compuestas del EuropASI ${ }^{25}$. Se utilizaron también el Symptom Check List $(\mathrm{SCL}-90-\mathrm{R})^{26}$, y el Beck Depresión Inventory ${ }^{27}$ para las medidas relacionadas con la psicopatología, y el Michigan Alcoholism Screening Test (MAST) ${ }^{28}$ para disponer una medida de la intensidad de los problemas con el alcohol. Finalmente se administró el Stages of Change Readiness Treatment Eagerness Scale (SOCRATES) ${ }^{29}$, para disponer de una medida relacionada con la motivación. Los instrumentos utilizados permiten establecer un diagnóstico multidisciplinar de la problemática adictiva, produciendo una información que facilita la planificación de una intervención individualizada para cada paciente. Además, todos ellos son instrumentos muy utilizados en investigación y permiten la comparación de resultados con otros estudios.

\section{Procedimiento}

Cualquier paciente que solicitaba iniciar tratamiento y cumplía con los criterios señalados, era incluido en el estudio. Las medidas basales se obtuvieron con una entrevista clínica y con los instrumentos de evaluación señalados anteriormente en las siguientes sesiones tras la admisión. En esta fase se descartó que la sintomatología psicopatológica fuera producida por la intoxicación, o por los efectos de la abstinencia. Además, se evaluó la abstinencia a la cocaína durante todo el tiempo de permanencia en el programa, testando a todos los 
pacientes semanalmente. El seguimiento se realizó desde la entrada en tratamiento hasta los seis primeros meses.

La variable dependiente fue el mes de abandono del tratamiento. Las variables independientes consideradas como posibles predictoras fueron las características sociodemográficas y las puntuaciones en los diferentes instrumentos: SCL90-R, BDI, MAST, SOCRATES y EuropASI.

\section{Análisis de datos}

Para el análisis de las características principales de los pacientes que componían la muestra se realizaron análisis descriptivos y de frecuencias. Para identificar las variables predictivas de la finalización prematura se utilizó un análisis factorial, un análisis cluster, y un análisis chi-square automatic interaction detection (CHAID). El análisis factorial ${ }^{30} \mathrm{es}$ un procedimiento multivariante de interdependencia que permite agrupar variables similares creando nuevas variables 0 "factores", y fue utilizado para agilizar el número de posibles variables predictoras. El análisis cluster ${ }^{30}$ permitió definir distintos perfiles de pacientes en función de las posibles variables predictoras. El análisis $\mathrm{CHAID}^{30}$ es un técnica multivariante de dependencia que permite conocer que variables son más influyentes y en qué medida lo son. Se utilizó el paquete estadístico SPSS-15 y un nivel de confianza del 95\%.

\section{RESULTADOS}

El porcentaje final de abandonos hasta los seis meses fue del 50,5\% (46 pacientes). En los tres primeros meses se concentraron la mayoría de abandonos (30,8\%), es decir 28 pacientes: 8 pacientes el primer mes $(8,8 \%), 12$ en el segundo mes $(13,2 \%)$ y 8 en el tercero $(8,8 \%)$. En los tres meses siguientes se producen los 18 abandonos restantes (19,7\%).

\section{Análisis factorial}

Utilizando todas las variables independientes mencionadas, el análisis factorial dio como resultado tres factores, o tres "variables resumen" que explicaban el 69,86\% de la varianza. La prueba de adecuación muestral KMO tuvo un valor de 0,87 y el test de esfericidad de Bartlett alcanzó un valor elevado $(815,51)$ siendo el nivel asociado de significación nulo ( $p$-valor $=0,00)$. Para poder interpretar mejor los factores o componentes, se realizó una rotación Varimax. Los resultados se presentan en la Tabla 1.

En función de sus cargas (superiores a 0,5 ), los factores o componentes, son interpretables denominándolos de la siguiente forma: Factor 1: "Psicopatología". En este factor aparecen los valores de las puntuaciones en el SCL-90-R. Factor 2: "Gravedad Adictiva". En este factor aparecen las valoraciones referidas a cuestionario MAST y las puntuaciones compuestas de drogas y alcohol. Factor 3: "Motivación". En este factor aparecen los valores de las puntuaciones en el cuestionario SOCRATES.
Tabla 1. Análisis Factorial

\begin{tabular}{|l|r|r|r|}
\hline \multirow{2}{*}{ Puntuaciones } & \multicolumn{3}{|c|}{ Componente } \\
\cline { 2 - 4 } & \multicolumn{1}{|c|}{1} & \multicolumn{1}{|c|}{2} & \multicolumn{1}{c|}{3} \\
\hline Centil Somatizaciones &, 707 &, 192 &,- 009 \\
Centil Obsesiones y compulsiones &, 877 &, 197 &, 174 \\
Centil Sensitividad interpersonal &, 810 &, 207 &, 074 \\
Centil Depresión &, 886 &, 174 &, 192 \\
Centil Ansiedad &, 917 &, 043 &, 092 \\
Centil Hostilidad &, 660 &, 281 &, 063 \\
Centil Ansiedad fóbica &, 628 &, 201 &,- 076 \\
Centil Ideación paranoide &, 816 &, 180 &, 065 \\
Centil Psicoticismo &, 898 &, 091 &,- 007 \\
Centil Indice global de severidad &, 964 &, 140 &, 120 \\
Centil Indice positivo de Malestar &, 946 &, 115 &, 082 \\
Puntuacion MAST &, 240 &, 796 &, 088 \\
Puntuación compuesta alcohol &, 064 &, 881 &,- 020 \\
Puntuación compuesta drogas &, 323 &, 659 &,- 040 \\
Socrates reconocimiento &, 191 &, 307 &, 715 \\
Socrates ambivalencia &, 151 &,- 007 &, 781 \\
Socrates primeros pasos &,- 077 &,- 149 &, 644 \\
\hline
\end{tabular}

\section{Análisis Cluster}

Se realiza un análisis cluster con cada uno de las componentes o "variables resumen" obtenidas con el análisis factorial. Este análisis nos ofrece distintos perfiles de pacientes. Se observa que en los tres factores aparecen dos grupos o conglomerados diferenciados. En los tres análisis de clasificación observamos que aparece un grupo con puntuaciones más altas y otro grupo con puntuaciones más bajas. La Tabla 2 muestra las valoraciones estándar de cada uno de estos dos grupos para los tres factores. Estas valoraciones debemos considerarlas como las valoraciones medias de los pacientes de cada grupo.

Tabla 2. Análisis Cluster

\begin{tabular}{|c|c|c|}
\hline \multirow{2}{*}{ Factor Psicopatología } & \multicolumn{2}{|c|}{ Conglomerado } \\
\hline & 1 & 2 \\
\hline Centil Somatizaciones & 39 & 78 \\
\hline Centil Obsesiones y compulsiones & 48 & 92 \\
\hline Centil Sensitividad interpersonal & 29 & 82 \\
\hline Centil Depresión & 28 & 91 \\
\hline Centil Ansiedad & 27 & 87 \\
\hline Centil Hostilidad & 38 & 78 \\
\hline Centil Ansiedad fóbica & 20 & 68 \\
\hline Centil Ideación paranoide & 23 & 80 \\
\hline Centil Psicoticismo & 23 & 91 \\
\hline Centil Indice global de severidad & 34 & 93 \\
\hline Centil Indice positivo de Malestar & 37 & 93 \\
\hline \multirow{2}{*}{ Factor Severidad Adictiva } & \multicolumn{2}{|c|}{ Conglomerado } \\
\hline & 1 & 2 \\
\hline Puntuacion MAST & 3 & 15 \\
\hline Composite score alcohol & ,2031 &, 4484 \\
\hline Composite score drogas &, 1902 & ,2442 \\
\hline \multirow{2}{*}{ Factor Motivación } & \multicolumn{2}{|c|}{ Conglomerado } \\
\hline & 1 & 2 \\
\hline Socrates reconocimiento & 33 & 24 \\
\hline Socrates ambivalencia & 16 & 14 \\
\hline Socrates primeros pasos & 36 & 35 \\
\hline
\end{tabular}




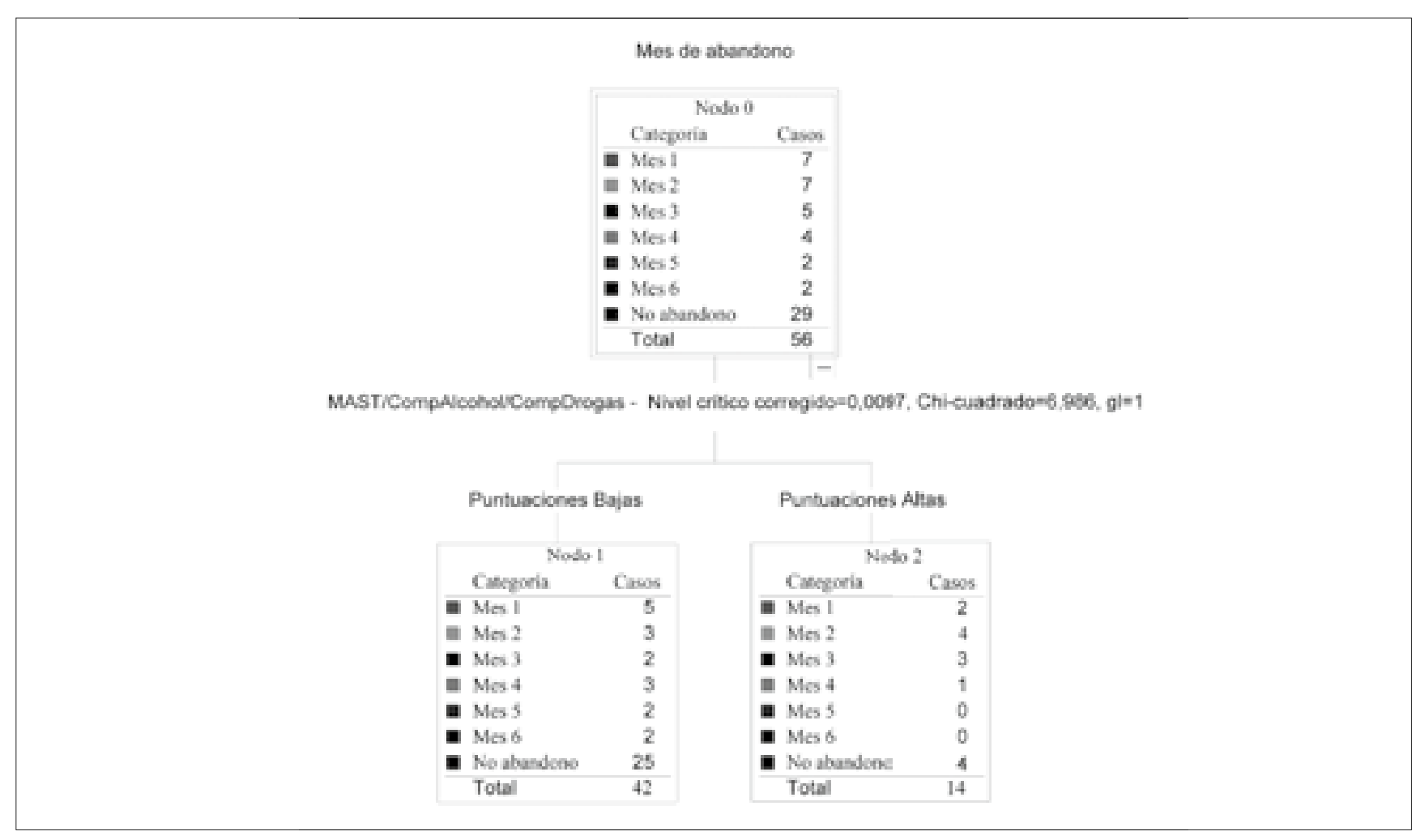

Figura 1. Análisis CHAID

Chi-square Automatic Interaction Detection Análisis (CHAID)

El análisis CHAID realizado tomando como variable dependiente el mes de abandono y como independientes los distintos perfiles de pacientes, mostró que los pacientes que más probabilidades tenían de permanecer en el tratamiento eran los que presentaban puntuaciones bajas en el factor "gravedad adictiva" ( $\left.\chi^{2}=6,986 ; g l=1 ; p=0,0097\right)$. No obstante, las valoraciones bajas en este factor no son totalmente determinantes para el no abandono, dado que el valor de la estimación del riesgo es de 0,4. En este análisis se trabaja con la posibilidad de tener tres niveles bajo el nodo raíz y con un número mínimo de casos de 10 en los nodos parentales y 5 en los nodos filiales, utilizando 56 de los 91 casos, por los valores perdidos, es decir, considerando como valores perdidos aquellos casos que no se ajustan, y no se han asignado a los perfiles obtenidos previamente. (Figura 1).

La mayoría de pacientes que no abandonan el tratamiento antes de los 6 meses ( 25 de 29) pertenecen al perfil de puntuaciones bajas en el factor "gravedad adictiva" (MAST, puntuación compuesta ASI-alcohol y puntuación compuesta ASI-drogas), es decir, tienen unas puntuaciones que rondan los valores de 3 (MAST), 0,20 (ASI-alcohol) y 0,19 (ASI-drogas), respectivamente. Se debe recordar que estas puntuaciones son las valoraciones estándar de los pacientes que pertenecen al perfil de pacientes con valoraciones bajas en dicho factor. Se observa también que en el perfil puntuaciones altas en el factor "severidad adictiva", se producen más abandonos que permanencias en el programa, y además, los abandonos se producen fundamentalmente en los tres primeros meses.

\section{DISCUSIÓN}

La investigación sobre las estrategias que mejoren la efectividad de las intervenciones que se utilizan en los problemas de abuso y adicción a la cocaína es una de las prioridades en el desarrollo de programas de tratamiento efectivo. Identificar las características clave asociadas a los motivos de abandono permite el desarrollo de estrategias más eficientes para mejorar las tasas de retención. En general, es importante evaluar las necesidades de tratamiento de los pacientes y los recursos disponibles en el momento de la admisión a tratamiento. Por ello, es relevante identificar qué estrategias específicas y qué servicios son adecuados para cada paciente.

En este estudio utilizamos distintos tipos de variables en el análisis predictivo (características sociodemográficas, consumo de alcohol y otras sustancias, indicadores de gravedad de la adicción, motivación, y estado afectivo), y se obtuvieron tres factores (gravedad adictiva, psicopatología y motivación), que explicaron una parte importante de la varianza del abandono. De entre estos factores, sólo el factor "gravedad adictiva" tuvo alguna capacidad predictiva, a pesar de que se utilizaron variables de tipo dinámico conjuntamente con otras de tipo estático, siguiendo recomendaciones de estudios previos ${ }^{32}$. Por otra parte, las variables relacionadas con la gravedad adictiva ya se han señalado como relevantes al considerar la evolución de los pacientes ${ }^{4,33}$ y la finalización prematura ${ }^{34,35}$. Nuestros resultados sugieren la importancia de evaluar detenidamente los aspectos relacionados con los patrones de uso no sólo de cocaína, sino también de alcohol. 
Tal y como se mencionó con anterioridad, el resultado final de un programa de tratamiento para adictos a cocaína está sujeto a la interacción dinámica de múltiples variables, por lo que se puede deducir que pueden aparecer una gran diversidad de patrones asociados al curso de la recuperación a largo plazo ${ }^{36}$. Cabría añadir además, que otro tipo de factores, como el tipo de tratamiento, el tiempo de espera hasta el inicio de la asistencia, la accesibilidad, las creencias de los pacientes sobre el tratamiento, la formación del personal y sus relaciones con los pacientes, y los recursos potenciales de éstos, serían otros factores importantes y facilitadores de cambio, como mecanismos clave en la retención y también en la recuperación de los pacientes. Estudios orientados a establecer posibles asociaciones entre los factores relacionados con el tratamiento y la retención, sería una de las líneas de investigación futuras sugeridas ${ }^{37}$.

Además de las limitaciones mencionadas, habría que añadir que puesto que los resultados se examinaron en un período de seis meses, sería conveniente estudiar la estabilidad de los predictores en un mayor período de seguimiento. Finalmente, el tamaño de la muestra debería ser ampliado en futuros trabajos para conseguir modelos de predicción más ajustados y generalizables, a partir de los cuáles puedan establecerse conclusiones más significativas.

La detección de pacientes que se encuentran en una situación de alto riesgo de incumplimiento del tratamiento puede ayudar a los clínicos a tomar medidas para modificar ese riesgo latente. La gravedad adictiva aunque no predice de forma totalmente determinante el abandono del tratamiento, sí que es importante al margen de los factores contextuales y los relacionados con el tratamiento. Ello podria implicar tomar decisiones de selección de pacientes en la admisión, una mayor preparación motivacional, mayor control e intensidad en el tratamiento, o la prestación de intervenciones coadyuvantes.

Quedan muchas interrogantes todavía por responder a la pregunta de porqué algunos pacientes y no otros abandonan los tratamientos de forma prematura. Una futura línea de investigación podría dirigirse a intentar establecer posibles relaciones entre las variables del paciente (dinámicas y estáticas), modalidades de tratamiento, el mantenimiento de la abstinencia o las recaídas continuadas, y la permanencia 0 no en los tratamientos. Dada la heterogeneidad de la población objeto de estudio, identificar "subgrupos" de pacientes en función de sus características y sus necesidades de tratamiento particulares podría ayudar en esta tarea.

\section{AGRADECIMIENTOS}

Este estudio forma parte de un proyecto de investigación financiado por la Delegación del Gobierno para el Plan Nacional sobre Drogas (Ministerio de Sanidad y Consumo de España) (Referencia MSC-06-01).

\section{REFERENCIAS}

1. Dutra L, Stathopoulou G, Basden S, Leyro T, Powers M, Otto M. A meta-analytic review of psychosocial interventions for substance use disorders. Am J Psychiatry 2008; 165: 179-187.

2. Veach L, Remley T, Kippers S, Sorg J. Retention predictors related to intensive outpatient programs for substance use disorders. Am J Drug Alcohol Abuse 2000; 26: 417-428.

3. Simpson D, Joe,G, Fletcher B, Hubbard R, Anglin D. A national evaluation of treatment outcomes for cocaine dependence. Arch Gen Psychiatry 1999; 56: 507-514.

4. Simpson D, Joe G, Broone K. A national 5-year follow-up of treatment outcomes for cocaine dependence. Arch Gen Psychiatry. 2002; 59: 538-544.

5. Hubbard R. Overview of 5-year follow-up outcomes in the drug abuse treatment outcome studies (DATOS). Journal of Substance Abuse Treatment 2003; 25: 125 - 134.

6. National Treatment Agency for Substance Misuse (NHS). National Evaluation of crack cocaine treatment and outcome study (NECTOS): A multicentre evaluation of dedicated crack treatment services. National Treatment Agency for Substance Misuse. UK. 2007.

7. Rowan-Szal G, Joe G, Simpson D. Treatment retention of crack cocaine users in a national sample of long term residential clients. Add Res \& Theory 2000; 8: 51-64.

8. Grella $C_{1}$ Hser $Y_{1}$ Hsieh S. Predictors of drug treatment re-entry following relapse to cocaine use in DATOS. J Subs Abuse Treat 2003; 25: 145-154.

9. Hser $Y$, Evans E, Huang D, Anglin D. Relationship between drug treatment services, retention, and outcomes. Psych Services 2004; 55: 767-774.

10. Ray G, Weisner C, Mertens J. Relationship between use of psychiatric services and five-year alcohol and drug treatment outcomes. Psych Services 2005; 56: 164-171.

11. Grella C, Stein J. Impact of program services on treatment outcomes of patients with co-morbid mental and substance use disorders, Psych Services 2006; 57: 1007-1015.

12. Meiers $P, B e s t D$. Programme factors that influence completion of residential treatment. Drug Alc Review 2006; 25: 349-355.

13. McMahon R. Substance abuse problems, psychiatric symptoms, and post-treatment status in MCMI psychopathology subgroups of cocaine dependent. Am J Drug Alc Abuse 2008; 34: 195-202.

14. Chi F, Weisner C. Nine-year psychiatric trajectories and substance use outcomes: an application of the group-based modeling approach. Eval Review. 2008; 32: 39-58.

15. Najavist L, Lester K. Gender differences in cocaine dependence. Drug Alc Dependen 2008; 97: 190-194.

16. Zhang Z, Gerstein D, Friedmann P. Patient satisfaction and sustained outcomes of drug abuse treatment. J Health Psych 2008; 13: 388-400.

17. Aharonovich $E_{1}$ Nunes $E_{1}$ Hasin D. Cognitive impairment, retention and abstinence among cocaine abusers in cognitivebehavioral treatment Drug Alc Depend 2003; 71: 207-211.

18. Barber J, Gallop R, Crits-Christoph P, Frank $A_{1}$ Thase $M$, Weiss $R$, Gibbons $M$. The role of therapist adherence, therapist competence, and the alliance in predicting outcome of individual drug counseling: Results from the National Institute of Drug Abuse Collaborative Cocaine Treatment Study. Psych Research 2006; 16: 229-240. 
19. Patkar A, Murray H, Mannelli P, Gottheil, P, Weinstein S, Vergare M. Pre-treatment measures of impulsivity, aggression and sensation seeking are associated with treatment outcome for African-American cocaine-dependent patients. J Add Diseases 2004; 23: 109-122.

20. Weiss $R$, Griffin $M$, Gallop $R$, Najavits $L$, Frank $A$, Crits-Christoph $P$, Thase M, Blaine J,Gastfriend D, Daley, D. The effect of 12-step self-help group attendance and participation on drug use outcomes among cocaine-dependent patients. Drug Alc Dependen 2005; 77: 177-184.

21. Carroll K, Nich C, Ball S. Practice makes progress? Homework assignments and outcome in treatment of cocaine dependence. J Cons Cli Psychology 2005; 73: 749-755.

22. Carroll K, Fenton L, Ball S, Nich C, Frankforter T, Shi J, Rounsaville B. Efficacy of disulfiram and cognitive behavior therapy in cocaine-dependent outpatients A randomized placebocontrolled trial Arch Gen Psychiatry 2004; 61: 264-272.

23. Garcia-Rodríguez 0 , Secades-Villa R, Álvarez $H$, Rio A, Fernández-Hermida J, Carballo J, Errasti J, Al-Halabi S. Efecto de los incentivos sobre la retención en un tratamiento ambulatorio para adictos a la cocaina. Psicothema 2007; 19: 134-139.

24. American Psychiatric Association. Diagnostic and Statistical Manual of Mental Disorders DSM-IV-TR, 4th edition. Washington: APA. 2000.

25. Kokkevi A, Hartgers C. European adaptation of a multidimensional assessment instrument for drug and alcohol dependence. Europ Add Research 1995; 1: 208-210.

26. Derogatis L, Lipman R, Covi L. SCL-90: An outpatient psychiatric rating scale-preliminary report. Psych Bulletin 1973; 9: 13-28.

27. Beck A, Ward C, Mendelson M, Mock J. Erbaugh J. An inventory for measuring depression. Arch Gen Psychiatry 1961; 4: 561-571.
28. Seltzer M. The Michigan Alcoholism Screening Test: The quest for a new diagnostic instrument. Am J Psychiatry 1971; 127: 1653-1658.

29. Miller P, Tonigan J. Assessing drinkers' motivation for change. The Stages of Change Readiness Treatment Eagerness Sacale (SOCRATES). Psych Addic Behav 1996; 10: 81-89.

30. Hair J, Anderson R, Tatham R, Black W. Multivariate analysis. Prentice Hall. 2001.

31. Lévy-Magin, J. Varela, J. Análisis multivariante para las ciencias sociales. Madrid. Prntice Hall. [Multivariate analysis for the social sciences]. 2003.

32. Stotts A, Mooney M, Sayre S, Novy M, Schmitz J, Grabowski J. Illusory predictors: Generalizability of findings in cocaine treatment retention research. Addic Behav 2007, 32: 28192836.

33. López $A$, Becoña $E$, Moratinos M, Montes A, Fernández I. Martínez $J$ y col. Evolución del tratamiento y consumo en personas con dependencia de la cocaina, a los doce meses, en función de la psicopatología asociada y el funcionamiento cotidiano. Adicciones 2007, 19: 251-266.

34. Gainey R, Wells E, Hawkins J, Catalano R. Predicting treatment retention among cocaine users. Subst Use Misuse 1993, 28: 487-505.

35. McCaul M. Predictors of outpatient treatment retention: patient versus substance use characteristics. Drug Alc Depend 2001, 62: 9-17.

36. Dennis M, Foss M, Scott C. An eight-year perspective on the relationship between the duration of abstinence and other aspects of recovery. Eval Review 2007, 31: 585-612. 2007, 31: 585-612.

37. Secades-Villa R, Magdalena Y. Predictores de la retención en una comunidad terapéutica para drogodependientes. Adicciones 2000, 12: 365-371. 